\title{
Voluntary motor drive: possible reduction in Tourette syndrome
}

\author{
C. A. Heise $\cdot$ V. Wanschura $\cdot$ B. Albrecht . \\ H. Uebel $\cdot$ V. Roessner $\cdot$ S. Himpel $\cdot$ W. Paulus $\cdot$ \\ A. Rothenberger $\cdot$ F. Tergau
}

Received: 7 May 2007/Accepted: 15 November 2007 / Published online: 15 January 2008

(C) The Author(s) 2008

\begin{abstract}
Electrophysiologically, Tourette syndrome (TS) is characterized by shortened cortical silent period (CSP), reflecting decreased motor inhibition. However, voluntary versus involuntary aspects of inhibitory functions in TS are not well understood. Hence, investigating voluntary motor drive (VMD) could help to elucidate this issue. A group of 14 healthy adolescents was compared with subjects of same age suffering from TS with $(N=6)$ and without $(N=6)$ presence of distal tics. Basic resting and active motor thresholds (RMT and AMT, respectively) as well as suprathreshold transcranial magnetic stimulation-conditioned RMT and AMT were determined during the CSP. The difference between AMT and RMT was considered as VMD quantum. No group-differences were found in RMT or AMT. Subjects with distal tics showed reduced VMD compared to healthy controls while patients without distal tics did not differ from controls. In the second half of CSP, patients with distal tics showed also diminished VMD compared to tic-patients without distal tics. The findings support the notion, that TS shows possible reduction of VMD and is associated with central motor threshold alterations confined to the very motor networks related to the tics observed.
\end{abstract}

C. A. Heise $(\square) \cdot$ B. Albrecht · H. Uebel · V. Roessner .

S. Himpel · A. Rothenberger

Child and Adolescent Psychiatry,

University of Göttingen, v. Siebold-Street 5,

37075 Göttingen, Germany

e-mail: cheise@gwdg.de

V. Wanschura $\cdot$ W. Paulus $\cdot$ F. Tergau

Department of Clinical Neurophysiology,

University of Göttingen, Robert-Koch-Strasse 40,

37075 Göttingen, Germany
Keywords Tourette syndrome - Adolescents · Transcranial magnetic stimulation .

Voluntary motor drive $\cdot$ Cortical silent period

\section{Introduction}

Tourette syndrome (TS) is a chronic neuropsychiatric disorder with a prevalence of about $1 \%$ in the general population. It is characterized by multiple motor and vocal tics, which have their onset in childhood. Tic disorders are often accompanied by other psychiatric problems like ADHD, OCD, ODD/CD, anxiety and depression, thus generating considerable negative impact on further development of the affected children (Rothenberger and Banaschewski 2005; Rothenberger et al. 2007).

While tics can be seen even during sleep and may be driven by thalamo-cortical oscillating dysrhythmia (Rothenberger et al. 2001; Leckman et al. 2006), they fulfill the criteria of involuntary movements only to some extent since many patients report sensorymotor phenomena that indicate the onset of tics and are able to suppress tics voluntarily for a short period of time (Leckman et al. 2001; Banaschewski et al. 2003). The latter requires further consideration of brain areas associated with volition, namely primary motor cortex, supplementary motor cortex and prefrontal motor cortex (Hallett 2001). Hence, the aim of this study was to further investigate the voluntary aspects in modulating inhibitory processes of the motor system. Several findings from earlier studies applying transcranial magnetic stimulation (TMS) support an inhibition deficiency model of tic disorders: reduced inhibitory activity in motor cortical structures or in afferent pathways from the basal ganglia to the motor cortical sites may lead to an imbalance of inhibiting and facilitating neuronal 
activity in cortico-striato-thalamo-cortical circuits that becomes manifest in tics (Moll et al. 2002; Leckman et al. 2006). Whereas in TS double-pulse-TMS measured cortical facilitation remained similar to healthy controls, cortical inhibition was reduced and cortical silent period (CSP) shortened (Ziemann et al. 1997; Gilbert et al. 2004, 2005).

To highlight the voluntary component of tics, we used a special measurement of TMS to explore and quantify its influence on motorcortex excitability. The excitability of the motor cortex increases during voluntary tonic contraction of the target muscle, which can be measured by determining the active motor threshold (AMT) and resting motor threshold (RMT). The difference between AMT and RMT indicates the magnitude and thus the voluntary amplification of descending cortico-muscular pathways, referred to as "voluntary motor drive" (VMD).

A previous study from our department with a sample of healthy adolescents and adult volunteers already evaluated the changes in the motor system during the CSP induced by an intense suprathreshold conditioning transcranial magnetic stimulus (intensity: two times CSP-threshold). Conditioned RMT (cRMT) showed an intensity-dependent period of elevation (lasting about $220 \mathrm{~ms}$ and was increased approximately $17 \%$ of maximum stimulator output above the unconditioned motor threshold), while conditioned AMT (cAMT) converged to the level found for cRMT. The separation of active and resting thresholds started in the late CSP and was nearly complete at its end. It was concluded that the CSP is largely due to suppression of VMD, while threshold elevation is a different inhibitory phenomenon that is of less importance for the generation of the CSP (Tergau et al. 1999). The aim of our investigation was to apply the method to adolescent patients with TS expecting to find that VMD is increased (probably acting as a trigger for tics) and threshold elevation during the CSP is less pronounced in Tourette patients especially when they suffer from distal tics due to an assumed deficit of cortical inhibition.

\section{Methods}

Right-handed European Caucasian subjects, aged between 10 and 15 years were included in the study. They belong to one of three subgroups:

1. Healthy control subjects without the presence of tics or any disorders regarding motor system $(\mathrm{C}, N=14)$.

2. Children fulfilling the diagnostic criteria for Tourette disorder according to the WHO's ICD-10 and APA's DSM-IV (American-Psychiatric-Association 1994) (group without distal tics, T, $N=6$ ).
3. Children fulfilling the diagnostic criteria for Tourette disorder according to the WHO's ICD-10 and APA's DSM-IV (group displaying distal tics, TdT, $N=6$ ).

Patients with TS were sequential outpatient referrals to the Department of Child and Adolescent Psychiatry/Psychotherapy at the University of Göttingen. They showed tics during the day of investigation. All members of patient groups were clinically assessed by a board-certified child and adolescent psychiatrist at the unit specializing in tic disorders, fulfilled DSM-IV criteria and showed medium tic severity. Several patients suffering from TS without distal tics were medicated with tiapride $(N=4)$ and one of them received additionally clomipramine while one patient received risperidone only, as well as two patients suffering from distal tics received tiapride of which one received additionally clonidine.

Experiments were performed according to the Declaration of Helsinki and approved by the ethics committee of the Medical Faculty of the University of Göttingen. All participants and their parents gave informed written consent and received no gratification except for reimbursement.

Handedness was ascertained according to the Oldfield scale of handedness (Oldfield 1971). All participants were right handed. Subjects were seated in a comfortable reclining chair. Surface electromyogram (EMG) was recorded from the right abductor digiti minimi muscle (ADM) with $\mathrm{Ag} / \mathrm{AgCl}^{-}$electrodes in a belly-to-tendon montage. The EMG was amplified, filtered (time constant: $10 \mathrm{~ms}$, low-pass filter: $2.5 \mathrm{kHz}$ ), digitized and fed into a desktop computer (sampling rate $5 \mathrm{kHz}$ ) using the NeuroScan 3.0 data collection and conditional averaging software. TMS was delivered through a figure-of-eight magnetic coil (each wing with a diameter of $7 \mathrm{~cm}$ ) connected, depending on the experiment, to one or two MAGSTIM 200 magnetic stimulators (maximum output $2 \mathrm{~T}$ each) via BiStim module (Magstim, Dyfed, Whitland, UK). The optimal position for activating the ADM was found by moving the coil in small steps around the presumed hand area of the left motor cortex and was defined as the site where stimuli of slightly suprathreshold intensity yielded the largest motor-evoked potentials (MEPs) in the target muscle. The inter stimulus interval (ISI) was at least 5 s. Data with artifacts due to tics were excluded.

\section{Determination of baseline motor threshold values}

Resting motor threshold was determined in the relaxed ADM under auditory EMG feedback control. Starting from a suprathreshold level, stimulus intensity was reduced in steps of $1 \%$ of maximum stimulator output and RMT was defined as the highest intensity yielding MEPs of peak-topeak amplitudes $<50 \mu \mathrm{V}$ in at least three out of six 
consecutive trials. AMT was similarly determined in the tonically activated ADM (10-20\% of maximum force). The CSP threshold was defined as the stimulator output intensity inducing a detectable inhibitory effect of no more than $20 \%$ reduction of voluntary EMG activity in the average of six consecutive rectified trials. To investigate the cortical changes in the motor system after TMS, a pulse of two-times SP-threshold intensity was used as a conditioning stimulus in the following experiments.

\section{Measurement of silent period duration}

With the conditioning stimulus, a CSP was induced in the active ADM. The CSP duration was calculated as the time from MEP-latency to the return to voluntary EMG activity. Twenty trials were performed, analyzed separately and the mean CSP duration values were computed. Both absolute and relative CSP duration were measured. While absolute CSP duration was defined as ending at the very first occurrence of voluntary EMG activity, the relative CSP duration was defined as ending when the rectified EMG again reached the pre-stimulus level.

\section{Assessment of voluntary motor drive}

To investigate the excitability of the cortico-muscular pathway, a second magnetic stimulus was used and conditioned motor thresholds (cRMT) were determined in the resting muscle at several ISIs (15-350 ms) after the conditioning stimulus. Since tonic activation of the target muscle increases the excitability of the cortico-muscular pathway, the difference between RMT and AMT is assumed to indicate the magnitude of VMD to the target muscle. A theoretical maximum possible VMD was calculated as the line integral area under the basal VMD between the first characteristic time point (first ISI when cRMT exceeds the RMT baseline level) and the fourth time point (end of aSP). To estimate the actual VMD during the CSP following the conditioning stimulus, cAMT and cRMT were determined in 5-20 ms steps after the conditioning stimulus up to $50 \mathrm{~ms}$ past the rSP. Additionally, individual characteristic time points (the first ISI when baseline level was exceeded, second at ISI with highest cRMT, third the ISI when cRMT recrosses baseline level, fourth at the end of the aCSP) were calculated and served as landmarks for determining the integral of VMD.

\section{Data analysis}

Since sample size was small and variances across groups were heterogeneous, the nonparametric Welch statistic was conducted to test for group differences on dependent variables (Welch 1947). In case of significance, additional nonparametric post-hoc Tamhane-T2 tests were performed. Significance level was set to $P<.05$, but since the sample size is small, trends with $P<.1$ are also reported.

\section{Results}

TMS basic variables

Groups were not found to be different on any of these parameters: Neither RMT $\left.\left(W_{(2,} 11.7\right)=1.9\right)$, AMT $\left(W_{(2,10.0)}=.2\right), \operatorname{aSP}\left(W_{(2,9.7)}=.2\right) \operatorname{nor} \operatorname{rSP}\left(W_{(2,10.8)}=.9\right)$ showed group-differences (Table 1).

\section{Voluntary motor drive}

As can be seen from Table 1, the assessment of theoretical maximum possible VMD revealed no group differences $\left(W_{(2,10.4)}=1.0\right)$. However, actual VMD was found to be different $\left(W_{(2,11.6)}=5.5^{*}\right)$, an effect present in the early as well as late half of CSP $\left(W_{(2,10.8)}=4.9 *\right.$ and $W_{(2,13.1)}=4.9^{*}$, respectively). Additional post-hoc analyses confirmed diminished VMD for the total as well as first and second halves of the CSP in subjects suffering from Tourette with distal tics, whereas in the second half only, even both groups with TS could be differentiated (all $P<.05$ ).

\section{Discussion}

Tics are neuropsychiatric phenomena of unique complexity with sensory motor phenomena before and/or after the tics (Banaschewski et al. 2003). Many patients describe that they voluntarily perform some of their tics to get relief from these sensory motor urges for a short period of time. Tics often can be voluntarily suppressed for seconds and minutes resulting in an increase of an unpleasant urge. However, some patients are not able to say whether their tics are voluntary or involuntary, so that tics may be reclassified as movement disorders in the transition zone between voluntary and involuntary.

To our knowledge this is the first study, which examined VMD in patients suffering from TS. The subgroup analysis revealed merely reduced mean VMD in the patient sample with distal tics supporting the assumption of Moll et al. (2006) that inhibitory neuronal deficits in tic disorders may be generalized during childhood but during adolescence are confined to the very motor networks related to the tics observed.

In addition, we found that VMD in TS seems to be basically similar to healthy controls, i.e., for non-tic related 
Table 1 Voluntary motor drive in patients with Tourette syndrome $(N=12)$ and healthy controls $(N=14)$

\begin{tabular}{|c|c|c|c|c|c|}
\hline Measure & $\begin{array}{l}\text { Controls (C) } \\
N=14 \\
\text { Mean (SD) }\end{array}$ & $\begin{array}{l}\text { Tourette without } \\
\text { distal tics } \\
\text { (T) } N=6 \\
\text { Mean (SD) }\end{array}$ & $\begin{array}{l}\text { Tourette with } \\
\text { distal tics } \\
\text { (TdT) } N=6 \\
\text { Mean }(\mathrm{SD})\end{array}$ & Welch-statistic & $\begin{array}{l}\text { Tamhane } \\
\text { post-hoc-tests }\end{array}$ \\
\hline \multicolumn{6}{|l|}{ Descriptives } \\
\hline Age (years) & $13.1(1.8)$ & $13.7(1.0)$ & $13.8(1.2)$ & $W_{(2,12.8)}=.7$ & - \\
\hline \multicolumn{6}{|l|}{ TMS basic variables } \\
\hline RMT & $52.3(6.7)$ & $55.9(5.7)$ & $49.7(4.9)$ & $W_{(2,11.7)}=1.9$ & - \\
\hline AMT & $36.8(4.3)$ & $36.5(3.9)$ & $34.9(6.4)$ & $W_{(2,10.0)}=.2$ & - \\
\hline Absolute CSP & $180.9(34.4)$ & $174.3(37.9)$ & $166.5(45.0)$ & $W_{(2,9.7)}=.2$ & - \\
\hline Relative CSP & $217.6(45.3)$ & $196.3(39.1)$ & $190.3(47.0)$ & $W_{(2,10.8)}=.9$ & - \\
\hline \multicolumn{6}{|l|}{$V M D$-integral } \\
\hline Possible VMD & $1,986(716)$ & $2,378(748)$ & $1,776(732)$ & $W_{(2,10.4)}=1.0$ & - \\
\hline Actual VMD & $1,055(582)$ & $1,087(465)$ & $593(173)$ & $W_{(2,11.6)}=5.5^{*}$ & $\mathrm{C}>\mathrm{TdT}^{*}$ \\
\hline Actual, first half VMD & $329(167)$ & $361(222)$ & $192(47)$ & $W_{(2,10.8)}=4.9^{*}$ & $\mathrm{C}>\mathrm{TdT}^{*}$ \\
\hline Actual, second half VMD & $726(461)$ & $725(250)$ & $401(153)$ & $W_{(2,13.1)}=4.9^{*}$ & $\mathrm{C}>\mathrm{TdT}^{+}, \mathrm{T}>\mathrm{TdT}^{+}$ \\
\hline VMD quotient & $.55(.27)$ & $.45(.12)$ & $.35(.10)$ & $W_{(2,14.0)}=3.0^{+}$ & $\mathrm{C}>\mathrm{TdT}^{+}$ \\
\hline
\end{tabular}

VMD: RMT - AMT (Tesla); VMD-integral: line-integral of the difference between RMT and AMT (Tesla ms); VMD quotient: possible VMD/ actual VMD

$V M D$ voluntary motor drive, TMS transcranial magnetic stimulation, $R M T$ resting motor threshold (\% maximum stimulator output, Tesla), AMT active motor threshold (\% maximum stimulator output, Tesla), CSP cortical silent period (ms)

$* P<.05$

$+P>.10$

muscle groups. However, most of the subjects suffering from TS without distal tics were treated with dopamine antagonists (like benzamides) whereas patients suffering from distal tics were mainly not medicated. Fortunately, this may not have confounded the findings since Ziemann et al. 1996 did not find an influence of either Haloperidol or Benzamides on AMT or RMT, i.e., the constituting base variables of VMD.

Although patients with other motor system disorders like Parkinson disease, Chorea Huntington, focal dystonia, thalamic lesions or lesions of the premotor cortex also were reported to show an altered CSP duration (Beradelli et al. 1996; Cantello et al. 1991; Haug et al. 1992; Valls-Solé et al. 1994; Rona et al. 1998; Schnitzler and Benecke 1994), there is some reason that the case of TS seems to be specific (Ziemann et al. 1997; Moll et al. 1999). Its neuronal disturbance is probably due to a primarily subcortical dysfunction in the striatum affecting the motor cortex through misguided brain oscillations (Leckman et al. 2006). The latter seems not to allow the patients with TS to inhibit their tic-related muscle groups, even in the case of a possibly reduced VMD as detected here. Hence, this study supports the notion that tics are principally involuntary movements (with reduced neuronal inhibition), which interact with voluntary aspects of behavior like frontal lobe compensatory mechanisms in tic suppression (Plessen et al. 2007) and VMD, which seems to play no role in tic facilitation.
It seems important to further investigate the interplay between the voluntary and involuntary aspects of TS in order to nurture the recent approaches in behavior therapy of TS (Döpfner and Rothenberger 2007).

Finally, it has to be stated, that the finding of a shortened CSP in TS could not be replicated in this study because of the differences in methods used. Since the main focus in our study was on examining the course of VMD after conditioning stimulus, a considerably higher stimulator intensity (approximately 200\% AMT) compared with previous studies (110-140\% AMT) addressing especially CSP-duration was used. There is a recent study showing that stimulus-intensity and duration of CSP are sigmoidally related with relative bottom-effects at very low and ceiling effects at high intensities (Kimiskidis et al. 2005). Thus, it may be more likely to detect group-differences at moderate stimulation intensities.

Open Access This article is distributed under the terms of the Creative Commons Attribution Noncommercial License which permits any noncommercial use, distribution, and reproduction in any medium, provided the original author(s) and source are credited.

\section{References}

American-Psychiatric-Association (1994) Diagnostic and statistical manual of mental disorders, 4th edn. American Psychiatric Association, Washington 
Banaschewski T, Woerner W, Rothenberger A (2003) Premonitory sensory phenomena and suppressibility of tics in Tourette syndrome: developmental aspects in children and adolescents. Dev Med Child Neurol 45:700-703

Beradelli A, Rona S, Inghilleri M, Manfredi M (1996) Cortical inhibition in Parkinson's disease. A study with paired magnetic stimulation. Brain 119(1):71-77

Cantello A, Gianelli M, Bettucci D, Civardi C, De Angelis MS, Mutani R (1991) Parkinson's disease rigidity: magnetic motor evoked potentials in a small hand muscle. Neurology 41(9):1449-1456

Döpfner M, Rothenberger A (2007) Behavior therapy in tic disorders with co-existing ADHD. Eur Child Adolesc Psychiatry 16(Suppl 1):i89-i99

Gilbert DL, Bansal AS, Sethuraman G, Sallee FR, Zhang J, Lipps T, Wassermann EM (2004) Association of cortical disinhibition with tic, ADHD, and OCD severity in Tourette syndrome. Mov Disord 19(4):416-425

Gilbert DL, Sallee FR, Zhang J, Lipps TD, Wassermann EM (2005) Transcranial magnetic stimulation-evoked cortical inhibition: a consistent marker of attention-deficit/hyperactivity disorder scores in Tourette syndrome. Biol Psychiatry 57:1597-1600

Hallett M (2001) Neurophysiology of tics. In: Cohen DJ, Goetz CG, Jankovic J (eds) Tourette syndrome. Lippincott Williams \& Wilkins, Philadelphia, pp 237-244

Haug BA, Schönle PW, Knobloch C, Köhne M (1992) Silent period measurement revives as a valuable diagnostic tool with transcranial magnetic stimulation. Electroencephalogr Clin Neurophysiol 85(2):158-160

Kimiskidis VK, Papagiannopoulos S, Sotirakoglou K, Kazis DA, Kazis A, Mills KR (2005) Silent period to transcranial magnetic stimulation: construction and properties of stimulus-response curves in healthy volunteers. Exp Brain Res 163:21-31

Leckman JF, Cohen DJ, Goetz CG, Jankovic J (2001) Tourette syndrome: pieces of the puzzle. In: Cohen DJ, Goetz CG, Jankovic J (eds) Tourette syndrome. Lippincott Williams \& Wilkins, Philadelphia, pp 369-390

Leckman JF, Vaccarino FM, Kalanithi PS, Rothenberger A (2006) Annotation: Tourette syndrome: a relentless drumbeat-driven by misguided brain oscillations. J Child Psychol Psychiatry 47:537-550

Moll GH, Wischer S, Heinrich H, Tergau F, Paulus W, Rothenberger A (1999) Deficient motor control in children with tic disorder: evidence from transcranial magnetic stimulation. Neurosci Lett 272:37-40

Moll GH, Heinrich H, Rothenberger A (2002) Transcranial magnetic stimulation in child psychiatry: disturbed motor system excitability in hypermotoric syndromes. Dev Sci 5(3):381-391
Moll GH, Heinrich H, Gevensleben H, Rothenberger A (2006) Tic distribution and inhibitory processes in the sensorimotor circuit during adolescence: a cross-sectional TMS study. Neurosci Lett 403(1-2):96-99

Oldfield RC (1971) The assessment and analysis of handedness: the Edinburgh inventory. Neuropsychologia 9(1):97-113

Plessen KJ, Royal JM, Peterson BS (2007) Neuroimaging of tic disorders with coexisting attention deficit hyperactivity disorder. Eur Child Adolesc Psychiatry 16(Suppl 1):i60-i70

Rona S, Beradelli A, Vacca L, Inghilleri M, Manfredi M (1998) Alterations of motor cortical inhibition in patients with dystonia. Mov Disord 13(1):118-124

Rothenberger A, Banaschewski T (2005) Tic-disorders. In: Gillberg C, Harrington R, Steinhausen HC (eds) A clinician's handbook of child and adolescent psychiatry. Cambridge University Press, Cambridge, pp 598-624

Rothenberger A, Kostanecka T, Kinkelbur J, Cohrs S, Woerner W, Hajak G (2001) Sleep and Tourette's syndrome. In: Cohen DJ, Jankovic J, Goetz C (eds) Tourette syndrome, advances in neurology. vol 85, Lippincott Williams and Wilkens, Philadelphia, pp 245-259

Rothenberger A, Roessner V, Banaschewski T, Leckman J (2007) Coexistence of tic disorders and attention-deficit/hyperactivity disorder-recent advances in understanding and treatment. Eur Child Adolesc Psychiatry 16(Suppl 9):1-4

Schnitzler A, Benecke R (1994) The silent period after transcranial magnetic stimulation is of exclusive cortical origin: evidence from isolated cortical ischemic lesions in man. Neurosci Lett 180(1):41-45

Tergau F, Wanschura V, Canelo M, Wischer S, Wassermann EM, Ziemann U, Paulus W (1999) Complete suppression of voluntary motor drive during the silent period after transcranial magnetic stimulation. Exp Brain Res 124(4):447-454

Valls-Solé J, Pascual-Leone A, Brasil-Neto JP, Cammarota A, McShane L, Hallett M (1994) Abnormal facilitation of the response to transcranial magnetic stimulation in patients with Parkinson's disease. Neurology 44(4):735-741

Welch BL (1947) The generalization of 'student's' problem when several different population variances are involved. Biometrika 34:28-35

Ziemann U, Lönnecker S, Steinhoff BJ, Paulus W (1996) Effects of antiepileptic drugs on motor cortex excitability in humans: a transcranial magnetic stimulation study. Ann Neurol 40:367-378

Ziemann U, Paulus W, Rothenberger A (1997) Decreased motor inhibition in Tourette disorder: evidence from transcranial magnetic stimulation. Am J Psychiatry 154:1277-1284 